Background The RCOPP was a national programme aimed at enabling older people to stay independent and well at home. Three of the interventions funded by the RCOPP in Glasgow City targeted COPD. This study aims to describe emergency admissions (EAs) for those with COPD in Glasgow City during the RCOPP.

Methods COPD EAs were defined using a primary diagnosis of COPD, while EAs for those with COPD were defined using any of the 6 diagnostic fields. Monthly standardised rates of emergency admission between April 2011 and March 2015 were calculated, for residents of Glasgow City aged 65 years+. Multilevel Zero-inflated Negative Binomial models for EAs nested by datazone adjusted for sex, 5 year agegroup, area-level deprivation (SIMD quintile), season, month and month squared. Relevant interventions were entered into the models, to test association with the two outcomes by time and location.

Results COPD EAs first rose, from April 2011 until October 2012, then fell until March 2015. When modelled, both month (RR for month 12 relative to month $1 \%$ and $95 \%$ $\mathrm{CI}=1.08 \quad(0.996,1.17))$ and month squared $(\mathrm{RR}=0.998$ $(0.996,0.999))$ were significant, with risk of admission falling below the baseline figures by October 2013 and continuing to reduce thereafter. EAs for COPD patients, however, had the opposite trend, first falling between April 2011 and February 2013, then rising until March 2015, although never reaching the level of April 2011. Under the model, month $(\mathrm{RR}=0.92$ $(0.87,0.97))$ and month squared $(R R=1.002(1.001,1.003))$ were both significant. However, this increase was only in the North East and North West sectors of Glasgow. EA for COPD patients reduced in the South, with particularly large reductions from July 2012. When included in the model, the Community Respiratory Team, in the North West, was associated with reductions in COPD EAs $(\mathrm{RR}=0.89(0.82,0.97))$ from the point of full staffing) and increases in EAs for COPD patients $(\mathrm{RR}=1.11(1.03,1.20))$. Two other projects were also potentially associated with increases in EAs for COPD.

Conclusion COPD EAs reduced from the end of 2012, in line with all-cause EAs shown previously. The timing and geography suggest these reductions may be due to the Community Respiratory project which helps people to manage their symptoms at home. EAs for COPD patients, however, increased from around the same time. This or another RCOPP service may have raised awareness of other health concerns or health more generally, increasing EAs for these patients.

\section{P40 EXPERIENCES AND ILLNESS PERCEPTIONS OF WORKING-AGE CARDIAC REHABILITATION ATTENDEES}

R Nutt*, G Ozakinci. School of Medicine, University of St Andrews, St Andrews, UK

\subsection{6/jech-2017-SSMAbstracts. 142}

Background Cardiac rehabilitation (CR) research often focuses on older individuals. With particular pressures faced by working-age individuals ( $<65$ years), such as work and family responsibilities, it is important to consider how this group engages with cardiac rehabilitation. We present here a synthesis of qualitative literature and initial results of a longitudinal qualitative study.

Methods We conducted thematic synthesis of studies (20062016) describing CR experiences of 18-65's. Synthesis was gender-sensitive and guided by Leventhal's Self-Regulation Model of Illness. Results informed development of the qualitative study.

The longitudinal qualitative study recruited participants aged $18-65$, following MI, from a Phase IV CR programme in Scotland. Semi-structured interviews occur at commencement and completion of the 12 week programme. Participants complete a questionnaire (Illness Perceptions QuestionnaireRevised and health behaviour questions) plus a family member is interviewed at both time points. Questionnaire data contextualises qualitative data (analysed using theoretical thematic analysis).

Results Review Nine studies were included. Heterogeneity existed in CR setting, participant numbers and gender. Thematic synthesis identified themes including illness perceptions, emotional representations and behaviour for illness control i.e. diet change. Some themes appear specific to the 'working-age' group. The influence of gender featured across all themes.

Study (At time of submission) Five participants and two family members interviewed, three baseline and follow-up, two baseline only. Participants are male, aged 41-61, all married and employed. Family members are female spouses. All participants had MI. Genetics or bad luck were often seen as cause of their MI, leading to limited behaviour change. Participants and family valued CR as a place of safety and reassurance due to monitoring and advice provided by staff. Participants identified themselves as fit and active, and therefore not 'old', suggesting recovering function/fitness may be particularly important for this age group. Following completion of CR, participants felt they could now exert themselves without causing their body or heart harm. Participants also described a lack of age-relevant support material that addressed issues like returning to work, family demands such as elderly parents, and exercise advice accounting for their greater pre-MI fitness.

Conclusion It is important to consider how working-age individuals experience $\mathrm{CR}$, as it impacts on engagement and behaviour. Existing literature regarding this group that also includes gender is limited and heterogeneous. Our study thus far indicates male working-age CR attendees and their family value the reassurance monitoring provides and allowing them to regain confidence in their bodies.

\section{P41 MENTAL HEALTH COMPETENCE IN ELEVEN YEAR OLDS AND ITS ASSOCIATION WITH POOR PHYSICAL HEALTH AND MENTAL WELL-BEING: FINDINGS FROM THE UK MILLENNIUM COHORT STUDY}

${ }^{1} \mathrm{E}$ Rougeaux*, 'A Pearce, ${ }^{2} \mathrm{~J}$ Deighton, ${ }^{1} \mathrm{C}$ Law, ${ }^{1} \mathrm{~S}$ Hope. ${ }^{1}$ Great Ormond Street Institute of Child Health, UCL, London, UK; ${ }^{2}$ Evidence Based Practice Unit, UCL and the Anna Freud Centre, London, UK

\subsection{6/jech-2017-SSMAbstracts. 143}

Background Positive mental health (not simply absence of mental disorder) may hold potential for fostering resilience and reducing risks of poor health and well-being. However, it is challenging to define and measure. One promising conceptualisation is mental health competence (MHC), comprising age-relevant developmental tasks and abilities. To date MHC has not been investigated in UK children.

Methods We developed a measure of MHC in the UK Millennium Cohort Study (MCS) ( 18000 children born 20002002) using seven items addressing learning skills and prosocial behaviours, reported by mothers at age $11 \quad(n=12082)$. 
Latent class analysis (LCA) was used to identify classes of MHC, and children were assigned to the class they had the highest probability of belonging to. We investigated associations between MHC classes and child's physical health (healthy weight, overweight or obese, based on measured heights and weights, using International Obesity Task Force cut-offs; maternal report of unintentional injuries since age 7 [none, 1, 2+]) and poor mental well-being (low self-esteem [SE] and low life-satisfaction [LS] reported by the child; maternal report of child's emotional problems [EP], all dichotomised at the bottom decile of scores). Relative risk ratios (aRRR, 95\% CI) and odds ratios (aORs, 95\% CI) were used to examine three-category and binary outcomes respectively, adjusting for potential confounding. Survey weights accounted for sample design and attrition. Analyses were undertaken in Stata/SE 13.1.

Results Four classes were identified (ranging from high to low $\mathrm{MHC})$ : "High learning skills and high prosocial behaviour" (37\%), "Moderate learning skills and high prosocial behaviour" (36\%), "Moderate learning skills and moderate prosocial behaviour" (19\%), and "Low learning skills and moderate prosocial behaviour" (8\%).

Risks of 2 +injuries were raised in "Moderate learning and high prosocial behaviour" (aRRR: 1.4 [95\%CI:1.2-1.7]) and "Low learning skills and moderate prosocial behaviour" (aRRR: 1.4 [95\%CI:1.1-1.9]) as compared to "High learning and high prosocial behaviour". Associations with MHC were absent or weak for single injury, overweight and obesity.

Compared to "High learning skills and high prosocial behaviour", odds of poor mental well-being were elevated for children from all other classes, with highest odds for "Low learning skills and moderate prosocial behaviour" (SE: aOR: 2.9 [95\%CI:2.3-3.6]; EP: aOR: 4.2 [95\%CI:3.4-5.1]; LS: aOR: 3.0 [95\%CI:2.4-3.7]).

Conclusion Lower MHC, using a composite measure developed in a representative sample of UK children, was associated with injuries, low self-esteem and life-satisfaction, and emotional problems, but not overweight and obesity. Identifying and promoting MHC at the population-level may provide an opportunity to improve health in children and young people.

\section{P42 "UNSEEN INJURIES": INVISIBILITY AND MENTAL ILLNESS IN THE ENGLISH WELFARE SYSTEM}

KJ Pybus* , K Pickett, C Lloyd. Department of Health Sciences, University of York, York, UK

\subsection{6/jech-2017-SSMAbstracts. 144}

Background Research focusing on the impact of current welfare reform in England has identified that people with mental illnesses experience pronounced difficulties when accessing social security compared to those with other health conditions. This includes a disproportionate risk of being sanctioned, problematic assessments resulting from a system designed to focus on physical capabilities and a greater likelihood of being viewed as a fraudulent claimant. The impact of individual policies is useful evidence, however the existence of disadvantage across several aspects of this system may imply the presence of underlying mechanisms which need to be understood if further marginalisation is to be prevented. The perspective of individuals with mental illness has been largely omitted from this discussion and may provide useful insights into these topics. The current study therefore aimed to explore the lived experiences of people with mental illness accessing housing and income benefits in England.

Methods Participants were recruited through five organisations offering support with social circumstances to people with mental illness in Leeds, England. Semi-structured interviews were completed with adults $(n=11)$ accessing housing and income benefits, living independently in the community. Convenience sampling in conjunction with organisation staff was employed to recruit participants. The sample was comprised of participants with the following mental health issues: depression $(\mathrm{n}=8)$, anxiety $(\mathrm{n}=5)$, post-traumatic stress disorder $(n=1)$, bi-polar affective disorder $(n=1)$, psychosis $(n=2)$, borderline personality disorder $(n=1)$ and substance misuse $(n=2)$ with several instances of co-morbidity. Data was interrogated using a six stage thematic analysis approach.

Results Accessing housing and income benefits can be problematic for people with mental illness due to the 'unseen' nature of these conditions. Barriers included being unable to provide evidence of illness to obtain financial support and the level of system flexibility in recognising the impact of mental health needs. Alongside this, participants encountered stigma from family, friends and the wider community rooted in the perceived validity of mental illness as a reason for claiming benefits. These themes were present across different types of mental health condition.

Conclusion These findings suggest that to create a social security system designed to be effective for people with a mental illness; changes are needed to the way in which mental illness is understood, assessed and monitored in this context. This study used a diverse but small sample in a localised setting and further research is needed to confirm these findings.

\section{P43 THE ASSOCIATION BETWEEN DEPRESSION AND SUBSEQUENT HYPERTENSION-A SYSTEMATIC REVIEW AND META-ANALYSIS}

R Prigge*, CA Jackson. Usher Institute of Population Health Sciences and Informatics, The University of Edinburgh, Edinburgh, UK

\subsection{6/jech-2017-SSMAbstracts. 145}

Background The possible causal association between depression and cardiovascular disease might be partly explained by an increased risk of hypertension. Several epidemiological studies have investigated the role of depression in the development of hypertension but the evidence is inconclusive. A previous systematic review of these studies has a number of shortcomings, including inappropriate pooling of different effect measures and lack of inclusion of all relevant studies. Additional primary studies have also been published since this earlier review was completed. Our aim was to identify, critically appraise, and synthesise evidence on the association between depression and subsequent hypertension.

Methods We performed a systematic electronic search in PsycINFO, Medline, and EMBASE to identify cohort or longitudinal studies reporting on the risk of hypertension among participants with versus without depressive symptoms and/or clinical depression. We restricted our search to articles published in English. We extracted information on study characteristics, methodology, and results using customised data extraction forms and assessed study quality using the SIGN checklist for cohort studies. We used Stata 14 to perform 